PROCEEDINGS OF THE

AMERICAN MATHEMATICAL SOCIETY

Volume 132, Number 8, Pages 2339-2349

S 0002-9939(04)07343-5

Article electronically published on March 25, 2004

\title{
PERIODIC SOLUTIONS OF NONLINEAR IMPULSIVE DIFFERENTIAL INCLUSIONS WITH CONSTRAINTS
}

\author{
TIZIANA CARDINALI AND RAFFAELLA SERVADEI
}

(Communicated by Carmen C. Chicone)

\begin{abstract}
In this paper we obtain the existence of periodic solutions for nonlinear "invariance" problems monitored by impulsive differential inclusions subject to impulse effects.
\end{abstract}

\section{INTRODUCTION}

The impulsive differential equations appear to represent a natural framework for mathematical modelings of several real world phenomena. For instance, systems with impulse effects have applications in physics, in biotechnology, in industrial robotics, in radiotechnology, in pharmacokinetics, in population dynamics, in ecology, in optimal control, in the study of microorganism reproduction, in economics, in production theory, and so on. The qualitative investigation of impulsive differential equations began in 1960 with the work of Mil'man and Myshkis (see 8]). The possibility of wide practical applications of impulsive differential equations explains the still growing interest of many authors in the investigation of these equations in recent years and the publication of monographs about this subject by Samoilenko and Perestyuk (see [11]), Lakshmikantham, Bainov and Simeonov (see [7]), and Bainov and Simeonov (see [1]). In the recent past, attention has been given to impulsive differential inclusions, and interesting results concerning the existence of periodic solutions for first-order impulsive differential inclusions have appeared: see, for instance, the papers by Watson (see [12]) and by Benchohra, Henderson, and Ntouyas (see [2]). But, unlike Benchohra, Henderson and Ntouyas, in [12] Watson studies an impulsive "invariance" problem. The fundamental tools to prove existence results for noninvariance problems are essentially fixed point arguments, nonlinear alternative, degree theory, or iterative methods combined with upper and lower solutions.

In this note we study the existence of periodic solutions for the following impulsive "invariance" problem:

$$
\begin{cases}x^{\prime}(t) \in F(t, x(t)) & \text { a.e. } t \in[0, T] \backslash\left\{t_{1}, \ldots, t_{p}\right\}, \\ x\left(t_{k}^{+}\right)=x\left(t_{k}\right)+I_{k}\left(x\left(t_{k}\right)\right) & \text { for any } k \in\{1, \ldots, p\}, \\ x(0)=x(T), & \end{cases}
$$

Received by the editors February 14, 2003 and, in revised form, April 29, 2003.

2000 Mathematics Subject Classification. Primary 34A37, 34A60, 34B15.

Key words and phrases. Impulsive differential inclusions and equations, canonical domain, Bouligand contingent cone, lower and upper semicontinuity of set-valued maps. 
where $\bar{\Omega} \subset \mathbb{R}^{N}, N \geq 1$, is a canonical domain, $F:[0, T] \times \bar{\Omega} \longrightarrow \mathcal{P}\left(\mathbb{R}^{N}\right)$ is a setvalued map, $0=t_{0}<t_{1}<\cdots<t_{p+1}=T, I_{k}: \bar{\Omega} \longrightarrow \mathbb{R}^{N}$ is an impulse function for any $k \in\{1, \ldots, p\}$, and $x\left(t^{+}\right)=\lim _{s \rightarrow t^{+}} x(s)$.

This problem is important in the case that one needs to study systems for which the state is required to stay in a set that may be constant. The pioneering work of Nagumo (see [9]) illustrates that the resolution of an "invariance" problem needs a tangential condition involving some kind of tangential approximation to the constraint set. More precisely, Nagumo proved that, for a continuous function $f: \mathbb{R}^{N} \longrightarrow \mathbb{R}^{N}$, a necessary and sufficient condition for the differential equation $x^{\prime}=f(x)$ a.e. on $\mathbb{R}^{N}$ to leave $K$ invariant is that $f(x) \in T_{K}(x)$ for all $x \in K$, where $T_{K}(x)$ denotes the Bouligand contingent cone to $K$ at $x \in K$.

In this paper we obtain existence results for the impulsive "invariance" problem $(P)$ (see Theorems 3.1 and 3.2). As in [12, in our proof we use the result due to Hristova and Bainov concerning the existence of a periodic solution for impulsive differential equations (see 3), together with an approximation argument. Our Theorems 3.1 and 3.2 extend the existence result proved by Watson (see Remarks 3.1 and 3.2 and, moreover, improve the Hristova - Bainov existence theorem in the case of "invariance" problems involving impulsive differential equations.

\section{Preliminaries}

Let $\Omega \subset \mathbb{R}^{N}, N \geq 1$, be a domain with boundary $\partial \Omega$, and let us denote by $\bar{\Omega}=\Omega \cup \partial \Omega$ its closure and by int $\bar{\Omega}$ the interior of $\bar{\Omega}$. We say that $\bar{\Omega}$ is a canonical domain (see [3] ) if $\Omega$ is bounded, convex and there exists a finite family of real-valued continuously differentiable maps $\left\{\Phi_{i}\right\}_{i \in\{1, \ldots, q\}}$ such that the following conditions hold:

$$
\begin{aligned}
& \bar{\Omega}=\bigcap_{i=1}^{q}\left\{x \in \mathbb{R}^{N}: \Phi_{i}(x) \leq 0\right\} ; \\
& \text { if there exist } x_{0} \in \partial \Omega \text { and } i \in\{1, \ldots, q\} \text { such that } \Phi_{i}\left(x_{0}\right)=0, \\
& \quad \text { then } \nabla \Phi_{i}\left(x_{0}\right) \neq 0 .
\end{aligned}
$$

Let us note that a compact interval of $\mathbb{R}$ is a canonical domain.

Let $\bar{\Omega}$ be a canonical domain defined by the family $\left\{\Phi_{i}\right\}_{i \in\{1, \ldots, q\}}$, and let us define $\alpha(x)=\left\{i \in\{1, \ldots, q\}: \Phi_{i}(x)=0\right\}$, for any $x \in \partial \Omega$, and

$$
P_{\bar{\Omega}}(x)= \begin{cases}\left\{y \in \mathbb{R}^{N}:\left\langle\nabla \Phi_{i}(x), y\right\rangle \leq 0, \forall i \in \alpha(x)\right\} & \text { if } x \in \partial \Omega, \\ \mathbb{R}^{N} & \text { if } x \in \Omega,\end{cases}
$$

where $\langle\cdot, \cdot\rangle$ is the scalar product in $\mathbb{R}^{N}$.

We recall the notion of Bouligand contingent cone to $\bar{\Omega}$ at $x \in \bar{\Omega}$ :

$$
T_{\bar{\Omega}}(x)=\left\{y \in \mathbb{R}^{N}: \liminf _{\lambda \rightarrow 0^{+}} \frac{\rho(x+\lambda y, \bar{\Omega})}{\lambda}=0\right\},
$$

where $\rho(z, \bar{\Omega})=\inf _{v \in \bar{\Omega}}\|z-v\|$. We note that $P_{\bar{\Omega}}(x)=T_{\bar{\Omega}}(x)$, for any $x \in \bar{\Omega}$ (see [12], Remark 1.3).

Moreover, we recall the definition of normal cone to $\bar{\Omega}$ at $x \in \bar{\Omega}$ :

$$
N_{\bar{\Omega}}(x)=\left\{x^{*} \in \mathbb{R}^{N}:\left\langle x^{*}, x\right\rangle=\sup _{z \in \bar{\Omega}}\left\langle x^{*}, z\right\rangle\right\} .
$$


The following property holds:

$$
y \in T_{\bar{\Omega}}(x) \Longleftrightarrow\left\langle x^{*}, y\right\rangle \leq 0, \forall x^{*} \in N_{\bar{\Omega}}(x) .
$$

Let $F:[0, T] \times \bar{\Omega} \longrightarrow \mathcal{P}\left(\mathbb{R}^{N}\right)$ be a set-valued map. $F$ is said to be lower semicontinuous (l.s.c.) in $(\bar{t}, \bar{x}) \in[0, T] \times \bar{\Omega}$ if, for any open set $A \subseteq \mathbb{R}^{N}$ such that $A \cap F(\bar{t}, \bar{x}) \neq \emptyset$, there exists a neighborhood $U$ of $(\bar{t}, \bar{x})$ such that $A \cap F(t, x) \neq \emptyset$, for any $(t, x) \in U \cap([0, T] \times \bar{\Omega})$. $F$ is said to be upper semicontinuous (u.s.c.) in $(\bar{t}, \bar{x}) \in[0, T] \times \bar{\Omega}$ if, for any open set $A \subseteq \mathbb{R}^{N}$ such that $F(\bar{t}, \bar{x}) \subseteq A$, there exists a neighborhood $U$ of $(\bar{t}, \bar{x})$ such that $F(t, x) \subseteq A$, for any $(t, x) \in U \cap([0, T] \times \bar{\Omega})$.

In this work we consider the following impulsive periodic "invariance" problem:

$$
\begin{cases}x^{\prime}(t) \in F(t, x(t)) & \text { a.e. } t \in[0, T] \backslash\left\{t_{1}, \ldots, t_{p}\right\}, \\ x\left(t_{k}^{+}\right)=x\left(t_{k}\right)+I_{k}\left(x\left(t_{k}\right)\right) & \text { for any } k \in\{1, \ldots, p\}, \\ x(0)=x(T), & \end{cases}
$$

where $\bar{\Omega} \subset \mathbb{R}^{N}, N \geq 1$, is a canonical domain, $F:[0, T] \times \bar{\Omega} \longrightarrow \mathcal{P}\left(\mathbb{R}^{N}\right)$ is a setvalued map, $0=t_{0}<t_{1}<\cdots<t_{p+1}=T, I_{k}: \bar{\Omega} \longrightarrow \mathbb{R}^{N}$ is an impulse function for any $k \in\{1, \ldots, p\}$, and $x\left(t^{+}\right)=\lim _{s \rightarrow t^{+}} x(s)$.

Definition 2.1. A solution of problem $(\mathcal{P})$ is a function $x:[0, T] \longrightarrow \bar{\Omega}$, absolutely continuous in the closed interval $\left[0, t_{1}\right]$ and in the interval $\left.] t_{k}, t_{k+1}\right]$ for any $k \in$ $\{1, \ldots, p\}$, such that

$$
\begin{array}{ll}
x^{\prime}(t) \in F(t, x(t)) & \text { a.e. } t \in[0, T] \backslash\left\{t_{1}, \ldots, t_{p}\right\} ; \\
x\left(t_{k}^{+}\right)=x\left(t_{k}\right)+I_{k}\left(x\left(t_{k}\right)\right) & \text { for any } k \in\{1, \ldots, p\} ;
\end{array}
$$

and

$$
x(0)=x(T) .
$$

To prove our existence results for problem $(\mathcal{P})$ we need the following theorem due to Hristova and Bainov ([3], Theorem 2) in the version proposed by Watson (12], Remarks 1.1 and 1.2, p. 397):

Theorem 2.1. Let $\bar{\Omega} \subset \mathbb{R}^{N}, N \geq 1$, be a canonical domain defined by the family $\left\{\Phi_{i}\right\}_{i \in\{1, \ldots, q\}}$, and let $f: \mathbb{R} \times \bar{\Omega} \longrightarrow \mathbb{R}^{N}$ and $I_{k}: \bar{\Omega} \longrightarrow \mathbb{R}^{N}, k \in \mathbb{Z}$, be functions such that

(B1) $\quad f$ is continuous in $\mathbb{R} \times \bar{\Omega}$;

(B2) $\exists T>0$ such that $f(t+T, x)=f(t, x), \forall(t, x) \in \mathbb{R} \times \bar{\Omega}$;

(B3) $\left\langle\nabla \Phi_{i}(x), f(t, x)\right\rangle \leq 0, \forall(t, x) \in \mathbb{R} \times \partial \Omega, \forall i \in \alpha(x)$;

(B4) $\quad I_{k}$ is continuous in $\bar{\Omega}$;

(B5) $\exists L>0:\|f(t, x)-f(t, y)\| \leq L\|x-y\|, \forall t \in \mathbb{R}, \forall x, y \in \bar{\Omega}$;

(B6) $\exists h \in \mathbb{N}: t_{k+h}=t_{k}+T, I_{k+h}(x)=I_{k}(x), \forall x \in \bar{\Omega}$;

(B7) $x+I_{k}(x) \in \bar{\Omega}, \forall x \in \bar{\Omega}$.

Then, the system of impulsive differential equations

$$
\begin{cases}x^{\prime}(t)=f(t, x(t)) & \text { a.e. } t \in \mathbb{R} \backslash\left\{t_{k}: k \in \mathbb{Z}\right\}, \\ x\left(t_{k}^{+}\right)=x\left(t_{k}\right)+I_{k}\left(x\left(t_{k}\right)\right) & \text { for any } k \in \mathbb{Z},\end{cases}
$$

has at least one $T$-periodic solution lying in $\bar{\Omega}$.

In this paper we also need the following theorem by Papageorgiou (see [10], Theorem 3.1). 
Theorem 2.2. Let $(M, \Sigma, \mu)$ be a complete, $\sigma$-finite measure space, $X$ a separable Banach space and $r \in\left[1, \infty\left[\right.\right.$. If $f_{n}, f \in L_{X}^{r}(M)$, for any $n \in \mathbb{N}, f_{n}(\cdot) \stackrel{w}{\longrightarrow} f(\cdot)$ in $L_{X}^{r}(M)$ and, for $\mu$-a.e. $t \in M$, there exists a nonempty, weakly-compact subset $G(t)$ of $X$ such that $f_{n}(t) \in G(t)$, then

$$
\begin{gathered}
f(t) \in \overline{\mathrm{co}} \mathrm{w}-\lim _{n \rightarrow \infty}\left\{f_{n}(t)\right\} \quad \text { for } \mu \text {-a.e. } t \in M, \\
\text { where } \mathrm{w}-\varlimsup_{n \rightarrow \infty}\left\{f_{n}(t)\right\}=\left\{x \in X: x=\mathrm{w}-\lim _{k \rightarrow \infty} f_{n_{k}}(t),\left(f_{n_{k}}(t)\right)_{k} \subseteq\left(f_{n}(t)\right)_{n}\right\} .
\end{gathered}
$$

\section{MAin Results}

In this section we state and prove our existence results on periodic solutions for the impulsive problem $(\mathcal{P})$.

Theorem 3.1. Let $\bar{\Omega} \subset \mathbb{R}^{N}, N \geq 1$, be a canonical domain such that int $\bar{\Omega} \neq \emptyset$, and let $F:[0, T] \times \bar{\Omega} \longrightarrow \mathcal{P}\left(\mathbb{R}^{N}\right)$ be a set-valued map such that the following conditions hold:

$(H 1) \quad F(t, x)$ is nonempty, convex, and closed, $\forall(t, x) \in[0, T] \times \bar{\Omega}$;

(H2) $F(t, \cdot)$ is u.s.c. in $\bar{\Omega}$, a.e. on $[0, T]$;

(H3) $\quad F$ is l.s.c. in $[0, T] \times \bar{\Omega}$;

(H4) $\quad F(t, x) \cap T_{\bar{\Omega}}(x) \neq \emptyset, \forall(t, x) \in[0, T] \times \bar{\Omega}$;

(H5) $\exists \gamma \in L^{\infty}([0, T])$ such that $\|F(t, x)\| \leq \gamma(t)$ a.e. on $[0, T], \forall x \in \bar{\Omega}$;

and let $I_{k}: \bar{\Omega} \longrightarrow \mathbb{R}^{N}, k \in\{1, \ldots, p\}$, be an impulse function such that

(B4) $I_{k}$ is continuous in $\bar{\Omega}$;

(B7) $x+I_{k}(x) \in \bar{\Omega}, \forall x \in \bar{\Omega}$.

Then, there exists a solution for the impulsive differential problem $(\mathcal{P})$.

Proof. Let $\left(\delta_{n}\right)_{n \in \mathbb{N}}, 0<\delta_{n} \leq 1$ for any $n \in \mathbb{N}$, be a sequence such that $\lim _{n \rightarrow \infty} \delta_{n}=0$ and, for any $n \in \mathbb{N}$, let $\hat{F}_{n}:[0, T] \times \bar{\Omega} \longrightarrow \mathcal{P}\left(\mathbb{R}^{N}\right)$ be a set-valued map defined as follows:

$$
\hat{F}_{n}(t, x)=F(t, x)+\delta_{n} B(0,1), \forall(t, x) \in[0, T] \times \bar{\Omega},
$$

where $B(0,1)$ is the ball of center 0 and radius 1 .

First of all, for a fixed $n \in \mathbb{N}$, we want to show that the set-valued map $\hat{F}_{n}(\cdot, \cdot) \cap$ $T_{\bar{\Omega}}(\cdot)$ is l.s.c. in $[0, T] \times \bar{\Omega}$. Since int $\bar{\Omega} \neq \emptyset$ yields $\operatorname{int} T_{\bar{\Omega}}(x) \neq \emptyset, \forall x \in \bar{\Omega}$ (see [5], p. 281), by using (H4), we have that $\hat{F}_{n}(t, x) \cap \operatorname{int} T \bar{\Omega}(x) \neq \emptyset, \forall(t, x) \in[0, T] \times \bar{\Omega}$. Moreover, (H3) and Proposition 2.59 of [4] imply that $\hat{F}_{n}$ is l.s.c. in $[0, T] \times \bar{\Omega}$. By Proposition 5.35 of [4] the set-valued map $(t, x) \longmapsto T_{\bar{\Omega}}(x),(t, x) \in[0, T] \times \bar{\Omega}$, is l.s.c. in $[0, T] \times \bar{\Omega}$ and has convex values. So, by Proposition 2.54 of [4, we can deduce that $\hat{F}_{n}(\cdot, \cdot) \cap T_{\bar{\Omega}}(\cdot)$ is l.s.c. in $[0, T] \times \bar{\Omega}$. Taking into account that the set-valued map $\hat{F}_{n}(\cdot, \cdot) \cap T_{\bar{\Omega}}(\cdot)$ has nonempty, convex and closed values, from Michael's selection theorem we can say that there exists a continuous selection $g_{n}:[0, T] \times \bar{\Omega} \longrightarrow \mathbb{R}^{N}$ for the set-valued map $\hat{F}_{n}(\cdot, \cdot) \cap T_{\bar{\Omega}}(\cdot)$ such that

$$
g_{n}(t, x) \in \hat{F}_{n}(t, x) \cap T_{\bar{\Omega}}(x), \forall(t, x) \in[0, T] \times \bar{\Omega} .
$$

Now, by Lemma 1 of Lasota and Yorke (see [6]), we have that there exists a locally Lipschitz function $\hat{g}_{n}:[0, T] \times \bar{\Omega} \longrightarrow \mathbb{R}^{N}$ such that

$$
\left\|g_{n}(t, x)-\hat{g}_{n}(t, x)\right\| \leq \delta_{n} \xi, \forall(t, x) \in[0, T] \times \bar{\Omega},
$$

where $\xi$ is a positive constant such that

$$
\xi \leq 1 \quad \text { and } \quad \xi\left\|x^{*}\right\| \leq\left\langle x^{*}, x\right\rangle, \forall x^{*} \in N_{\bar{\Omega}}(x) .
$$


At this point, let us define $f_{n}:[0, T] \times \bar{\Omega} \longrightarrow \mathbb{R}^{N}$ as follows:

$$
f_{n}(t, x)=\hat{g}_{n}(t, x)-\delta_{n} x, \forall(t, x) \in[0, T] \times \bar{\Omega} .
$$

Now we prove that

$$
f_{n}(t, x) \in T_{\bar{\Omega}}(x), \forall(t, x) \in[0, T] \times \bar{\Omega} .
$$

In order to obtain (7), let us fix $(t, x) \in[0, T] \times \bar{\Omega}$ and $x^{*} \in N_{\bar{\Omega}}(x)$. Taking into account (6), (3), (5), (4) and (1), we have that

$$
\begin{aligned}
& \left\langle x^{*}, f_{n}(t, x)\right\rangle=\left\langle x^{*}, \hat{g}_{n}(t, x)-\delta_{n} x\right\rangle \\
& =\left\langle x^{*}, g_{n}(t, x)\right\rangle+\left\langle x^{*}, \hat{g}_{n}(t, x)-g_{n}(t, x)\right\rangle-\delta_{n}\left\langle x^{*}, x\right\rangle \\
& \leq\left\|x^{*}\right\|\left\|\hat{g}_{n}(t, x)-g_{n}(t, x)\right\|-\delta_{n} \xi\left\|x^{*}\right\| \\
& \leq \delta_{n} \xi\left\|x^{*}\right\|-\delta_{n} \xi\left\|x^{*}\right\|=0,
\end{aligned}
$$

and so (7) is proved.

Recalling that $f_{n}$ is Lipschitz in $[0, T] \times \bar{\Omega}$ (being locally Lipschitz in the compact, convex set $[0, T] \times \bar{\Omega}$ ), Hristova and Bainov's theorem (see Theorem 2.1) implies that the impulsive periodic problem

$$
\left(\mathcal{P}_{n}\right) \quad \begin{cases}x^{\prime}(t)=f_{n}(t, x(t)) & \text { a.e. } t \in[0, T] \backslash\left\{t_{1}, \ldots, t_{p}\right\}, \\ x\left(t_{k}^{+}\right)=x\left(t_{k}\right)+I_{k}\left(x\left(t_{k}\right)\right) & \text { for any } k \in\{1, \ldots, p\}, \\ x(0)=x(T) & \end{cases}
$$

has a solution $x_{n}:[0, T] \longrightarrow \bar{\Omega}$, absolutely continuous in the closed interval $\left[0, t_{1}\right]$ and in the interval $\left.] t_{k}, t_{k+1}\right]$ for any $k \in\{1, \ldots, p\}$.

Let us define $y_{n}:[0, T] \longrightarrow \mathbb{R}^{N}$ as follows:

$$
y_{n}(t)= \begin{cases}x_{n}(t) & \text { if } t \in\left[0, t_{1}\right], \\ x_{n}(t)-\sum_{j=1}^{k} I_{j}\left(x_{n}\left(t_{j}\right)\right) & \text { if } \left.t \in] t_{k}, t_{k+1}\right], k \in\{1, \ldots, p\},\end{cases}
$$

for any $n \in \mathbb{N}$. Now, we prove that the sequence $\left(y_{n}\right)_{n \in \mathbb{N}}$ is equibounded in $[0, T]$. First of all, we observe that, for any $n \in \mathbb{N}$,

$$
x_{n}(t)= \begin{cases}x_{n}(0)+\int_{0}^{t} f_{n}\left(s, x_{n}(s)\right) d s & \text { if } t \in\left[0, t_{1}\right], \\ x_{n}(0)+\sum_{j=1}^{k} I_{j}\left(x_{n}\left(t_{j}\right)\right)+\int_{0}^{t} f_{n}\left(s, x_{n}(s)\right) d s & \text { if } \left.t \in] t_{k}, t_{k+1}\right], \\ & k \in\{1, \ldots, p\} .\end{cases}
$$

Then it follows that

$$
y_{n}(t)=x_{n}(0)+\int_{0}^{t} f_{n}\left(s, x_{n}(s)\right) d s,
$$

for all $t \in[0, T]$ and for any $n \in \mathbb{N}$. So, the fact that $\bar{\Omega}$ is bounded (this implies that there exists $R>0$ such that $\bar{\Omega} \subseteq B(0, R)$ ), (9), (6), (4), (3), (2), (H5) and the choice of $\left(\delta_{n}\right)_{n \in \mathbb{N}}$ yield

$$
\left\|y_{n}(t)\right\| \leq\left\|x_{n}(0)\right\|+\int_{0}^{t}\left\|f_{n}\left(s, x_{n}(s)\right)\right\| d s \leq R+K T,
$$

for all $t \in[0, T]$ and for any $n \in \mathbb{N}$, where $K=1+\xi+R+\|\gamma\|_{L^{\infty}([0, T])}$.

Now let us prove that the sequence $\left(y_{n}\right)_{n \in \mathbb{N}}$ is equicontinuous in $[0, T]$. Let us fix $t, s \in[0, T]$. By (9), (6), (44), (3), (2), $(H 5)$, the choice of $\left(\delta_{n}\right)_{n \in \mathbb{N}}$ and the 
boundedness of $\bar{\Omega}$ we have that

$$
\left\|y_{n}(t)-y_{n}(s)\right\|=\left\|\int_{s}^{t} f_{n}\left(z, x_{n}(z)\right) d z\right\| \leq K|t-s|,
$$

for any $n \in \mathbb{N}$; so $\left(y_{n}\right)_{n \in \mathbb{N}}$ is equicontinuous in $[0, T]$.

Then, by the Ascoli - Arzelà theorem, there exists a subsequence of $\left(y_{n}\right)_{n \in \mathbb{N}}$, which we again denote by $\left(y_{n}\right)_{n \in \mathbb{N}}$, such that

$$
y_{n} \longrightarrow y_{0} \text { uniformly on }[0, T] \text {. }
$$

By (8), (11) and (B4) it follows that there exists a subsequence of $\left(x_{n}\right)_{n \in \mathbb{N}}$, denoted again by $\left(x_{n}\right)_{n \in \mathbb{N}}$, such that

$$
x_{n} \longrightarrow x_{0} \text { uniformly on }[0, T] .
$$

We claim that $x_{0}$ is a solution of the impulsive differential inclusion $(\mathcal{P})$. The fact that $x_{n}$ solves problem $\left(\mathcal{P}_{n}\right)$, for any $n \in \mathbb{N}$, (12) and (B4) yield

$$
x_{0}(t) \in \bar{\Omega}, \forall t \in[0, T], x_{0}(0)=x_{0}(T),
$$

and

$$
\begin{aligned}
x_{0}\left(t_{k}^{+}\right) & =\lim _{t \rightarrow t_{k}^{+}} x_{0}(t)=\lim _{t \rightarrow t_{k}^{+}} \lim _{n \rightarrow \infty} x_{n}(t)=\lim _{n \rightarrow \infty} x_{n}\left(t_{k}^{+}\right) \\
& =\lim _{n \rightarrow \infty}\left[x_{n}\left(t_{k}\right)+I_{k}\left(x_{n}\left(t_{k}\right)\right)\right]=x_{0}\left(t_{k}\right)+I_{k}\left(x_{0}\left(t_{k}\right)\right),
\end{aligned}
$$

for any $k \in\{1, \ldots, p\}$. Now we prove that $x_{0}$ is absolutely continuous in the closed interval $\left[0, t_{1}\right]$ and in the interval $\left.] t_{k}, t_{k+1}\right]$ for any $k \in\{1, \ldots, p\}$. Actually, by (10), the sequence $\left(y_{n}\right)_{n \in \mathbb{N}}$ is equi-Lipschitz in $[0, T]$. So, by using (8), we can say that $\left(x_{n}\right)_{n \in \mathbb{N}}$ is equi-Lipschitz in $\left[0, t_{1}\right]$ and in $\left.] t_{k}, t_{k+1}\right]$, for any $k \in\{1, \ldots, p\}$. By passing to the limit as $n$ goes to infinity, we obtain that $x_{0}$ is Lipschitz in $\left[0, t_{1}\right]$ and in $\left.] t_{k}, t_{k+1}\right]$, for any $k \in\{1, \ldots, p\}$. It remains to show that $x_{0}^{\prime}(t) \in F\left(t, x_{0}(t)\right)$ a.e. $t \in[0, T] \backslash\left\{t_{1}, \ldots, t_{p}\right\}$. Without loss of generality, given a fixed $k \in\{1, \ldots, p\}$, we can suppose that $x_{n}$ and $x_{0}$ are continuous in $\left[t_{k}, t_{k+1}\right]$ by defining them in $t_{k}$ in this way:

$$
x_{n}\left(t_{k}\right)=x_{n}\left(t_{k}^{+}\right) \quad \text { and } \quad x_{0}\left(t_{k}\right)=x_{0}\left(t_{k}^{+}\right),
$$

for any $n \in \mathbb{N}$. By using the fact that $x_{n}$ is a solution of problem $\left(\mathcal{P}_{n}\right)$, for any $n \in \mathbb{N}$, (6), (4), (3), (2), (H5), the choice of $\left(\delta_{n}\right)_{n \in \mathbb{N}}$ and the boundedness of $\bar{\Omega}$ yield that the sequence $\left(x_{n}^{\prime}\right)_{n \in \mathbb{N}}$ is bounded in $L^{2}\left(\left[t_{k}, t_{k+1}\right]\right)$. So, there exist $v_{k} \in L^{2}\left(\left[t_{k}, t_{k+1}\right]\right)$ and a subsequence of $\left(x_{n}^{\prime}\right)_{n \in \mathbb{N}}$, denoted again by $\left(x_{n}^{\prime}\right)_{n \in \mathbb{N}}$, such that

$$
x_{n}^{\prime} \stackrel{w}{\longrightarrow} v_{k} \text { in } L^{2}\left(\left[t_{k}, t_{k+1}\right]\right) .
$$

Since $x_{n}$ is absolutely continuous in $\left[t_{k}, t_{k+1}\right]$, for any $n \in \mathbb{N}$ we have that

$$
x_{n}(t)-x_{n}\left(t_{k}\right)=\int_{t_{k}}^{t} x_{n}^{\prime}(s) d s,
$$

for all $t \in\left[t_{k}, t_{k+1}\right]$. By (12), (13), (14) and the absolute continuity of $x_{0}$ in $\left[t_{k}, t_{k+1}\right]$ it follows that $x_{0}^{\prime}=v_{k}$ a.e. on $\left[t_{k}, t_{k+1}\right]$. The same argument can be used to prove that there exists $v_{0} \in L^{2}\left(\left[0, t_{1}\right]\right)$ such that $x_{0}{ }^{\prime}=v_{0}$ a.e. on $\left[0, t_{1}\right]$. So

$$
x_{n}^{\prime} \stackrel{w}{\longrightarrow} x_{0}^{\prime} \text { in } L^{2}([0, T]) .
$$


Let us denote by $N \subset[0, T]$ the set such that $\mu N=0$ and hypotheses (H2) and (H5) are satisfied in $([0, T] \backslash N) \times \bar{\Omega}$. Now let us fix $t \in[0, T] \backslash N$ and $\epsilon>0$. By the choice of $\left(\delta_{n}\right)_{n \in \mathbb{N}}$, it follows that

$$
\exists n_{1}=n_{1}(\epsilon) \in \mathbb{N}: \forall n \geq n_{1} \delta_{n}<\epsilon .
$$

By using (H2), we can say that

(17) $\exists \rho=\rho\left(\epsilon, x_{0}(t)\right)>0: \forall x \in \bar{\Omega},\left\|x-x_{0}(t)\right\|<\rho, F(t, x) \subseteq F\left(t, x_{0}(t)\right)+B(0, \epsilon)$.

By (12), we have that

(18) $\exists n_{2}(\epsilon, t)=n_{2}\left(\rho\left(\epsilon, x_{0}(t)\right)\right): \forall n \geq n_{2}(\epsilon, t)\left\|x_{n}(s)-x_{0}(s)\right\|<\rho, \forall s \in[0, T]$.

Then, by (17) and (18), we obtain that

$$
F\left(t, x_{n}(t)\right) \subseteq F\left(t, x_{0}(t)\right)+B(0, \epsilon), \forall n \geq n_{2}(\epsilon, t) .
$$

Then, setting $n(\epsilon, t)=\max \left\{n_{1}(\epsilon), n_{2}(\epsilon, t)\right\}$, by (21), (16) and (19) we have that

$$
\hat{F}_{n}\left(t, x_{n}(t)\right) \subseteq F\left(t, x_{0}(t)\right)+B(0,2 \epsilon), \forall n \geq n(\epsilon, t) .
$$

Since $x_{n}$ is a solution for problem $\left(\mathcal{P}_{n}\right)$, for any $n \in \mathbb{N}$, (6), (3), (44) and the boundedness of $\bar{\Omega}$ imply

$$
\begin{aligned}
x_{n}^{\prime}(t) & =f_{n}\left(t, x_{n}(t)\right)=\hat{g}_{n}\left(t, x_{n}(t)\right)-\delta_{n} x_{n}(t) \\
& =g_{n}\left(t, x_{n}(t)\right)+\hat{g}_{n}\left(t, x_{n}(t)\right)-g_{n}\left(t, x_{n}(t)\right)-\delta_{n} x_{n}(t) \\
& \in \hat{F}_{n}\left(t, x_{n}(t)\right)+(\xi+R) \delta_{n} B(0,1)
\end{aligned}
$$

a.e. $t \in[0, T] \backslash\left\{t_{1}, \ldots, t_{p}\right\}$ and for any $n \in \mathbb{N}$. By using (21), (2), (H5) and the choice of $\left(\delta_{n}\right)_{n \in \mathbb{N}}$ and $\xi$, we have that

$$
\begin{aligned}
x_{n}^{\prime}(t) & \in F\left(t, x_{n}(t)\right)+\delta_{n} B(0,1)+(\xi+R) \delta_{n} B(0,1) \\
& \subseteq(\gamma(t)+2+R) B(0,1) \equiv G(t)
\end{aligned}
$$

a.e. $t \in[0, T] \backslash\left\{t_{1}, \ldots, t_{p}\right\}$ and for any $n \in \mathbb{N}$. Taking into account that $G(t)$ is a convex and weakly compact subset of $\mathbb{R}^{N}$, (15) and (22) allow us to deduce that (see Theorem 2.2)

$$
x_{0}^{\prime}(t) \in \overline{\mathrm{co}} \mathrm{w}-\varlimsup_{n \rightarrow \infty}\left\{x_{n}^{\prime}(t)\right\} \text { a.e. } t \in[0, T] .
$$

Now, by (16) and (21) it follows that

$$
x_{n}^{\prime}(t) \in \hat{F}_{n}\left(t, x_{n}(t)\right)+(\xi+R) B(0, \epsilon)
$$

a.e. $t \in[0, T] \backslash\left\{t_{1}, \ldots, t_{p}\right\}$, for any $n \geq n(\epsilon, t)$. Then (23) and (24) yield

$$
x_{0}^{\prime}(t) \in \overline{\mathrm{CO}} \mathrm{w}-\varlimsup_{n \rightarrow \infty}\left(\hat{F}_{n}\left(t, x_{n}(t)\right)+(\xi+R) B(0, \epsilon)\right)
$$

a.e. $t \in[0, T] \backslash\left\{t_{1}, \ldots, t_{p}\right\}$. So, by (25), (201) and (H1), we obtain that

$$
x_{0}^{\prime}(t) \in \overline{\mathrm{co}}\left(F\left(t, x_{0}(t)\right)+(2+\xi+R) B(0, \epsilon)\right)=F\left(t, x_{0}(t)\right)+(2+\xi+R) B(0, \epsilon)
$$

a.e. $t \in[0, T] \backslash\left\{t_{1}, \ldots, t_{p}\right\}$. So, the arbitrariness of $\epsilon$, (26) and (H1) yield

$$
x_{0}^{\prime}(t) \in F\left(t, x_{0}(t)\right) \text { a.e. } t \in[0, T] \backslash\left\{t_{1}, \ldots, t_{p}\right\} .
$$


Remark 3.1. We note that there exist set-valued maps satisfying the hypotheses of Theorem [3.1, but not the conditions of Theorem 2.1 due to Watson (see [12]). For example, we can consider the set-valued map $F:[0,1] \times[0,1] \longrightarrow \mathcal{P}(\mathbb{R})$ defined as follows:

$$
F(t, x)= \begin{cases}\{0\} & \text { if } t=0, x \in[0,1] \\ {[0,1]} & \text { if } t \in] 0,1], x \in[0,1] .\end{cases}
$$

Now, by using Theorem 3.1 we are able to prove the following result.

Theorem 3.2. Let $\bar{\Omega} \subset \mathbb{R}^{N}, N \geq 1$, be a canonical domain such that int $\bar{\Omega} \neq \emptyset$, and let $F:[0, T] \times \bar{\Omega} \longrightarrow \mathcal{P}\left(\mathbb{R}^{N}\right)$ be a set-valued map such that the following conditions hold:

(C1) $F(t, x)$ is nonempty, convex, and closed a.e. on $[0, T], \forall x \in \bar{\Omega}$;

(H2) $F(t, \cdot)$ is u.s.c. in $\bar{\Omega}$, a.e. on $[0, T]$;

(C2) $\quad \exists\left(F_{n}\right)_{n \in \mathbb{N}}, F_{n}:[0, T] \times \bar{\Omega} \longrightarrow \mathcal{P}\left(\mathbb{R}^{N}\right)$ such that

$(C 2.1) \quad F_{n}(t, x)$ is nonempty, convex, and closed, $\forall(t, x)$ $\in[0, T] \times \bar{\Omega}, \forall n \in \mathbb{N}$

$(C 2.2) \quad F_{n}$ is l.s.c. in $[0, T] \times \bar{\Omega}, \forall n \in \mathbb{N}$;

$(C 2.3) \quad F_{n}(t, x) \cap T_{\bar{\Omega}}(x) \neq \emptyset, \forall(t, x) \in[0, T] \times \bar{\Omega}, \forall n \in \mathbb{N}$;

(C2.4) $\exists \gamma \in L^{\infty}([0, T])$ such that $\left\|F_{n}(t, x)\right\| \leq \gamma(t)$

a.e. on $[0, T], \forall x \in \bar{\Omega}, \forall n \in \mathbb{N}$;

$(C 2.5) \quad$ a.e. $t \in[0, T], \forall \epsilon>0 \exists \bar{n}=\bar{n}(\epsilon, t) \in \mathbb{N}$ such that

$F_{n}(t, x) \subseteq F(t, x)+B(0, \epsilon), \forall n \geq \bar{n}, \forall x \in \bar{\Omega}$;

and let $I_{k}: \bar{\Omega} \longrightarrow \mathbb{R}^{N}, k \in\{1, \ldots, p\}$, be an impulse function such that

(B4) $\quad I_{k}$ is continuous in $\bar{\Omega}$;

(B7) $x+I_{k}(x) \in \bar{\Omega}, \forall x \in \bar{\Omega}$.

Then, there exists a solution for the impulsive differential problem $(\mathcal{P})$.

Proof. Let $\left(\delta_{n}\right)_{n \in \mathbb{N}}, 0<\delta_{n} \leq 1$ for any $n \in \mathbb{N}$, be a sequence such that $\lim _{n \rightarrow \infty} \delta_{n}=0$ and, for any $n \in \mathbb{N}$, let $\hat{F}_{n}:[0, T] \times \bar{\Omega} \longrightarrow \mathcal{P}\left(\mathbb{R}^{N}\right)$ be a set-valued map defined as follows:

$$
\hat{F}_{n}(t, x)=F_{n}(t, x)+\delta_{n} B(0,1), \forall(t, x) \in[0, T] \times \bar{\Omega} .
$$

In order to prove the existence of a continuous selection $g_{n}:[0, T] \times \bar{\Omega} \longrightarrow \mathbb{R}^{N}$ for the set-valued map $\hat{F}_{n}(\cdot, \cdot) \cap T_{\bar{\Omega}}(\cdot)$, we can apply the same arguments used in the first part of the proof of Theorem 3.1. Now let us consider the impulsive periodic problem

$$
\begin{cases}x^{\prime}(t)=g_{n}(t, x(t)) & \text { a.e. } t \in[0, T] \backslash\left\{t_{1}, \ldots, t_{p}\right\}, \\ x\left(t_{k}^{+}\right)=x\left(t_{k}\right)+I_{k}\left(x\left(t_{k}\right)\right) & \text { for any } k \in\{1, \ldots, p\}, \\ x(0)=x(T) . & \end{cases}
$$

By Theorem 3.1 there exists a solution $x_{n}:[0, T] \longrightarrow \bar{\Omega}$ for problem $\left(\mathcal{P}_{n}\right)$, for any $n \in \mathbb{N}$. Arguing as in the proof of Theorem 3.1 we obtain that

$$
x_{n} \longrightarrow x_{0} \text { uniformly on }[0, T] \text {, }
$$

where $x_{0}$ is a function, absolutely continuous in the closed interval $\left[0, t_{1}\right]$ and in the interval $\left.] t_{k}, t_{k+1}\right]$ for any $k \in\{1, \ldots, p\}$, such that

$$
\begin{gathered}
x_{0}(t) \in \bar{\Omega}, \forall t \in[0, T], x_{0}(0)=x_{0}(T), \\
x_{0}\left(t_{k}^{+}\right)=x_{0}\left(t_{k}\right)+I_{k}\left(x_{0}\left(t_{k}\right)\right), k \in\{1, \ldots, p\},
\end{gathered}
$$


and

$$
x_{n}^{\prime} \stackrel{w}{\longrightarrow} x_{0}^{\prime} \text { in } L^{2}([0, T]) .
$$

To prove that $x_{0}$ is a solution for the impulsive differential problem $(\mathcal{P})$, it remains to show that $x_{0}^{\prime}(t) \in F\left(t, x_{0}(t)\right)$ a.e. $t \in[0, T] \backslash\left\{t_{1}, \ldots, t_{p}\right\}$.

Let us denote by $N^{*} \subset[0, T]$ the set such that $\mu N^{*}=0$ and $(C 1),(H 2),(C 2.4)$ and $(C 2.5)$ are satisfied in $\left([0, T] \backslash N^{*}\right) \times \bar{\Omega}$. Now, let us fix $t \in[0, T] \backslash N^{*}$ and $\epsilon>0$. Hypothesis $(C 2.5)$ implies that

$$
\exists \bar{n}=\bar{n}(\epsilon, t) \in \mathbb{N}: \forall n \geq \bar{n}(\epsilon, t) F_{n}\left(t, x_{n}(t)\right) \subseteq F\left(t, x_{n}(t)\right)+B(0, \epsilon) .
$$

As in Theorem 3.1, by the choice of $\left(\delta_{n}\right)_{n \in \mathbb{N}},(H 2)$ and (28), we can say that there exists $\tilde{n}=\tilde{n}(\epsilon, t) \in \mathbb{N}$ such that

$$
\delta_{n}<\epsilon \quad \text { and } \quad F\left(t, x_{n}(t)\right) \subseteq F\left(t, x_{0}(t)\right)+B(0, \epsilon), \forall n \geq \tilde{n}(\epsilon, t) .
$$

Then, setting $n^{*}(\epsilon, t)=\max \{\bar{n}(\epsilon, t), \tilde{n}(\epsilon, t)\}$, by (30) and (31) we obtain that

$$
\hat{F}_{n}\left(t, x_{n}(t)\right) \subseteq F\left(t, x_{0}(t)\right)+B(0,3 \epsilon), \forall n \geq n^{*}(\epsilon, t) .
$$

Since $x_{n}$ is a solution for problem $\left(\mathcal{P}_{n}\right)$ and $g_{n}$ is a selection for $\hat{F}_{n}(\cdot, \cdot) \cap T_{\bar{\Omega}}(\cdot)$, for any $n \in \mathbb{N}$, (27), $(C 2.4)$ and the choice of $\left(\delta_{n}\right)_{n \in \mathbb{N}}$ imply

$$
x_{n}^{\prime}(t) \in \hat{F}_{n}\left(t, x_{n}(t)\right)=F_{n}\left(t, x_{n}(t)\right)+\delta_{n} B(0,1) \subseteq B(0, \gamma(t)+1) \equiv G(t)
$$

a.e. $t \in[0, T] \backslash\left\{t_{1}, \ldots, t_{p}\right\}$ and for any $n \in \mathbb{N}$. Taking into account that $G(t)$ is a convex and weakly compact subset of $\mathbb{R}^{N},(29)$ and (33) allow us to deduce that (see Theorem [2.2)

$$
x_{0}^{\prime}(t) \in \overline{\mathrm{co}} \mathrm{w}-\varlimsup_{n \rightarrow \infty}\left\{x_{n}^{\prime}(t)\right\} \text { a.e. } t \in[0, T] .
$$

Since $\left\{x_{n}^{\prime}(t)\right\} \subseteq \hat{F}_{n}\left(t, x_{n}(t)\right)$ a.e. $t \in[0, T] \backslash\left\{t_{1}, \ldots, t_{p}\right\}$, for any $n \in \mathbb{N}$, by (34) it follows that

$$
x_{0}^{\prime}(t) \in \overline{\mathrm{co}} \mathrm{w}-\varlimsup_{n \rightarrow \infty} \hat{F}_{n}\left(t, x_{n}(t)\right)
$$

a.e. $t \in[0, T] \backslash\left\{t_{1}, \ldots, t_{p}\right\}$. Then, by (35), (32) and $(C 1)$, we obtain that

$$
x_{0}^{\prime}(t) \in \overline{\mathrm{co}}\left(F\left(t, x_{0}(t)\right)+B(0,3 \epsilon)\right)=F\left(t, x_{0}(t)\right)+B(0,3 \epsilon)
$$

a.e. $t \in[0, T] \backslash\left\{t_{1}, \ldots, t_{p}\right\}$. So, by the arbitrariness of $\epsilon$, (36) and $(C 1)$, we can conclude that $x_{0}$ is a solution of problem $(\mathcal{P})$.

Remark 3.2. Let us remark that Theorem 3.2 improves Theorem 3.1 (and so it extends the result due to Watson (see Remark 3.1)). It is obvious that, if $F$ satisfies all the hypotheses of Theorem 3.1, we can take $F_{n}=F$, for any $n \in \mathbb{N}$. Moreover, there exist set-valued maps $F$ satisfying the hypotheses of Theorem 3.2 that are not l.s.c. For example, we can consider the set-valued map $F:[0,2] \times[0,1] \longrightarrow \mathcal{P}(\mathbb{R})$ defined as follows:

$$
F(t, x)= \begin{cases}{[-1,0]} & \text { if } t \in[0,1[, x \in[0,1] \\ {[-1,1]} & \text { if } t=1, x \in[0,1], \\ {[-1,2]} & \text { if } t \in] 1,2], x \in[0,1]\end{cases}
$$


$F$ is not l.s.c. in $[0,2] \times[0,1]$, but it satisfies hypotheses $(C 1)$ and $(H 2)$ of Theorem 3.2. Moreover, we can construct the sequence $\left(F_{n}\right)_{n \in \mathbb{N}}, F_{n}:[0,2] \times[0,1] \longrightarrow \mathcal{P}(\mathbb{R})$, in this way:

$$
F_{n}(t, x)= \begin{cases}{\left[-1, t^{n}\right]} & \text { if } t \in[0,1], x \in[0,1] \\ {[-1,2]} & \text { if } t \in] 1,2], x \in[0,1]\end{cases}
$$

for any $n \in \mathbb{N}$.

This sequence satisfies conditions $(C 2.1),(C 2.2),(C 2.3),(C 2.4)$ and, since it converges to $F,(C 2.5)$.

We also want to observe that in Theorem 3.2 we do not need that the sequence $\left(F_{n}\right)_{n \in \mathbb{N}}$ converges to $F$. Indeed, the sequence $\left(\tilde{F}_{n}\right)_{n \in \mathbb{N}}, \tilde{F}_{n}:[0,2] \times[0,1] \longrightarrow \mathcal{P}(\mathbb{R})$ defined by

$$
\tilde{F}_{n}(t, x)= \begin{cases}{\left[-1, \frac{1}{n}\right]} & \text { if } t \in[0,1], x \in[0,1], \\ {[-1,2]} & \text { if } t \in] 0,1], x \in[0,1],\end{cases}
$$

for any $n \in \mathbb{N}$, does not converge to the set-valued map $F$ defined as in (37), but it satisfies all the hypotheses of Theorem 3.2 .

Remark 3.3. Let us note that there exist u.s.c., but not l.s.c., set-valued maps $F$ saisfying the hypotheses of Theorem 3.2 For example, we can consider the setvalued map $F:[0,2] \times[0,1] \longrightarrow \mathcal{P}(\mathbb{R})$ defined as follows:

$$
F(t, x)= \begin{cases}{[-1,0]} & \text { if } t \in[0,1[, x \in[0,1], \\ {[-1,1]} & \text { if } t \in[1,2], x \in[0,1] .\end{cases}
$$

In this case we can construct the sequence $\left(F_{n}\right)_{n \in \mathbb{N}}, F_{n}:[0,2] \times[0,1] \longrightarrow \mathcal{P}(\mathbb{R})$, in this way:

for any $n \in \mathbb{N}$.

$$
F_{n}(t, x)= \begin{cases}{\left[-1, \frac{1}{n}\right]} & \text { if } t \in[0,1], x \in[0,1], \\ {[-1,1]} & \text { if } t \in] 1,2], x \in[0,1]\end{cases}
$$

Remark 3.4. Finally, we observe that there exist set-valued maps satisfying the hypotheses of Theorem 3.2 that are neither l.s.c. nor u.s.c. For example, we can consider the set-valued map $F$ defined as in (37).

\section{REFERENCES}

[1] D. D. Bainov and P. S. Simeonov: Systems with impulsive effect. Stability, theory and applications, Ellis Horwood Series in Mathematics and its Applications, Ellis Horwood, Chichester, 1989. MR 90i:93082

[2] M. Benchohra, J. Henderson, and S. K. Ntouyas: On a periodic boundary value problem for first order impulsive differential inclusions, Dynam. Systems Appl. 10 (2001), 477-488. MR 2002i:34015

[3] S. G. Hristova and D. D. Bainov: Existence of periodic solutions of nonlinear systems of differential equations with impulse effect, J. Math. Anal. Appl. 125 (1987), 192-202. MR 88f:34055

[4] S. Hu and N. S. Papageorgiou: Handbook of multivalued analysis, Kluwer, Dordrecht, 1997. MR 98k:47001

[5] S. Hu and N. S. Papageorgiou: On the topological regularity of the solution set of differential inclusions with constraints, J. Differential Equations 107 (1994), 280-289. MR 94m:34036

[6] A. Lasota and J. A. Yorke: The generic property of existence of solutions of differential equations in Banach space, J. Differential Equations 13 (1973), 1-12. MR 49:770

[7] V. Lakshmikantham, D. D. Bainov, and P. S. Simeonov: Theory of impulsive differential equations, World Scientific, Series in Modern Appl. Math., 6, Singapore, 1989. MR 91m:34013

[8] V. D. Mil'man and A. D. Myshkis: On the stability of motion in the presence of impulses, Sibirsk. Mat. Zh. 1 (2) (1960), 233-237. (Russian) MR 23:A3325 
[9] M. Nagumo: Über die Lage der Integralkurven gewöhnlicker Differentialgleichungen, Proc. Phys.-Math. Soc. Japan, 24 (1942), 551-559. MR 7:381e

[10] N. S. Papageorgiou: Convergence theorems for Banach space valued integrable multifunctions, Internat. J. Math. Math. Sci. 10 (3) (1987), 433-442. MR 88i:28019

[11] A. M. Samoilenko and N. A. Perestyuk: Differential equations with impulse effect, World Scientific, Singapore (1995) (Visca Skola, Kiev (1987), in Russian).

[12] P. J. Watson: Impulsive differential inclusions, Nonlinear World 4 (1997), 395-402. MR 2000e:34018

Department of Mathematics and Computer Science, University of Perugia, via VanVitelli 1, Perugia 06123, Italy

E-mail address: tiziana@dipmat.unipg.it

Department of Mathematics, University of Roma 'Tor Vergata', via della Ricerca Scientifica, Roma 00133, Italy

E-mail address: servadei@mat.uniroma2.it 